\title{
Operational effectiveness of future land combat teams: model conceptualisation
}

\author{
$\underline{\text { M.K. Richmond }}^{\text {a }}$, M.S. Gary ${ }^{\text {b }}$, T. Cao ${ }^{\text {a }}$, D. Blumson ${ }^{\text {a }}$, L. Kosowski ${ }^{\text {a }}$, K. Hock ${ }^{\text {a }}$, S. Elsawah ${ }^{\text {c }}$ \\ and N. Kempt ${ }^{\text {a }}$ \\ ${ }^{a}$ Defense Science \& Technology Group, Edinburgh South Australia, ${ }^{b}$ School of Management \& Governance, \\ UNSW Sydney, New South Wales, ${ }^{c}$ School of Engineering, UNSW Canberra, Australian Capital Territory \\ Email: matthew.richmond@dst.defence.gov.au
}

\begin{abstract}
Modern land combat teams conduct a wide range of operations, including high intensity conflict, counter insurgency, humanitarian assistance, plus many more. Operating environments also span a diverse range, including (for example) urban, jungle and littoral, against a variety of different adversaries and threat levels. In the future operating environment, the complexity of operations will only increase. Consequently, future combat teams must be designed for a broad scenario space. Although there has been extensive operations research modelling of land combat operations, in general it focuses on high-fidelity detailed modelling or highly aggregated models. Neither of these two classes of land combat models enable rapid analysis of the operational effectiveness of future combat team performance. Further, most land combat models have tended to focus on representing kinetic factors and neglected non-kinetic and cognitive/social factors which have become critical to land warfare outcomes. We are interested in the ability to conduct medium-fidelity analysis of different proposed combat team designs ${ }^{1}$ and particularly to evaluate the potential impacts of new and emerging technologies, in order to inform: (i) technology research \& development priorities, (ii) insights for Land programs on potential technological impacts, (iii) explore the interplay between kinetic and non-kinetic effects, soft and hard factors, on combat effectiveness and (iv) to identify promising force designs to examine further using more detailed close loop simulation. We suggest that to achieve rigorous and responsive assessment of operational effectiveness (i.e., military utility) of incorporating new technologies into future combat teams across a range of (future) operational environments, including both hard and soft factors, requires a new class of model(s) conceptualised at a level of aggregation somewhere between the high-fidelity and highly aggregated models cited above.
\end{abstract}

This paper reports on the initial conceptualisation of a model to address this gap. The model focuses on representing the generic tactical level effects that a combat team must achieve to prosecute its required activities (e.g., assault, ambush, defend, etc.). By taking a generic approach to representing tactical level effects in the baseline model reported here, it is designed to be readily improved to consider a broad scenario space (as part of future work). We assume the generic effects and tasks required in future tactical land combat will persist, but the performance requirements will be influenced by the future operational environment and threat levels. Future combat team designs will be critical to how well the effects and activities can be performed.

We have focussed initially on a system dynamics (SD) modelling approach as it lends itself to the representation of a combat team at different levels of fidelity, ranging from highly abstract to very detailed. SD modelling also supports the representation of both quantitative and qualitative effects. We start by building a Baseline SD model representing a tactical land warfare combat team employing conventional (i.e., existing) technologies. A combat team force element is small enough to model quickly and to discriminate the impacts of specific technologies on operational effectiveness, but large enough to include combined arms capabilities and so key interdependencies between capabilities can be investigated. The Baseline model will provide the foundation for subsequent efforts to evaluate the impacts of future technologies compared with a combat team enabled with current technology, as well as "what-if" sensitivity analysis of more qualitative inputs such as morale and exploration of the scenario space. We discuss the next steps for further development of the baseline model and acknowledge the utility of incorporating other modelling techniques into a multi-method approach.

\footnotetext{
${ }^{1}$ By combat team design we mean an organisational structure, key personnel and equipment, and concepts describing how the force will operate focusing on the differences from current doctrine.
}

Keywords: Land combat, combined arms conceptual model, operational effectiveness evaluation, military utility assessment of emerging technology 


\section{INTRODUCTION}

While extensive operations research modelling has focussed on analysing land combat operations, much of that work involves either high-fidelity, detailed modelling at the entity level (e.g., representing each armoured vehicle and each soldier for the Blue and Red forces) (Balogh and Harless, 2003; Bowley, Castles, and Ryan, 2003; Finlay, 2013) or highly-aggregated models that abstract well-above the tactical battlefield including Force level simulations such as Advanced Warfighting Simulation (AWARS) (Gach and Sanders, 2015) or counter-insurgency models of multi-year military campaigns (Anderson Jr, 2011).

Other notable approaches include:

- High-fidelity combat simulation models such as COMBATXX1 (Chau, Gill, and Grieger, 2017). COMBATXXI is an entity-level, high-fidelity stochastic combat simulation. The model represents forceon-force scenarios down to the individual soldier, combat vehicles system components such as firepower and sensors, and tactical decision making and environmental factors such as terrain and light levels. Such high-fidelity modelling is resource intensive and normally focuses on a limited set of vignettes.

- Bayesian Network (BN) models that examine operational effectiveness using causal relationships and conditional probabilities. This approach yields important insights in some contexts, but feedback loops and dynamic behaviour are not well represented by BN (Nguyen and Cao, 2019).

- The Method to Analyse Relations between Variables using Enrich Loop (MARVEL) (Veldhuis et al., 2015) integrates problem structuring methods (such as SODA - Strategic Options Development and Analysis, and causal loop diagrams) with simulation techniques. It is designed for policy intervention analysis for defence problems with limited quantitative data availability. Although MARVEL has significantly influenced the authors' thinking, it includes a simplified application of system dynamics which was intended for problems for which a full Stock and Flow Diagram model is not feasible or desirable (Veldhuis et al. 2015).

In summary, prior combat modelling approaches are high-fidelity, therefore having significant overheads, and/or do not adequately account for the impact of less tangible variables such as "morale" and "Will to Fight" in warfighting. Our conceptual model seeks to fill this gap.

This paper builds directly on the previous work of the DSTG Team to build qualitative causal models of tactical Land warfare (Gaidow, Kosowski, and O’Hanlon, 2021; Kempt, Kosowski, and Weinberg, 2021). These previous efforts included a unified close combat model that represents cross-domain combined arms operations at the combat team level of detail, and which embodies Army doctrine that defeating the will of the enemy to fight is the overarching goal. The inputs required to generate the close combat causal map were sourced from the military doctrine of Australia, UK, Canada, and US. The find, fix, strike and exploit framework provided a strong basis from which to identify the core capabilities, their interactions, and the generation of effects against an opposing force. Further, the doctrine clearly described the role of non-lethal and other non-traditional capabilities. The causal model was designed to help assess technological, capability and structural variances in combat team designs.

These causal modelling efforts were necessary because prior close combat models (see for example: Pincombe, Pincombe, and Pearce, 2010a; Speight and Rowland, 2006) emphasise attrition-based (loss exchange ratio mindset), as opposed to the cross-domain effects necessary to support analysis of future warfighting concepts. For example, many prior models adopt an attrition-based, differential equation approach using the well-known Lanchester's Equations (Pincombe, Pincombe, and Pearce, 2010b). This approach models attrition based on force loss ratio and has greatly influenced military combat modelling for many years. However, winning a conflict involves either total annihilation or continued attrition until adversary force numbers reach a predetermined, low level. While perhaps applicable to outdated forms of warfare, this underlying assumption must be adapted to represent modern warfare that relies heavily on discrepancies of terrain, communications, sensors, weapon technologies, soldier capabilities, morale and, most importantly, will to fight. While we understand that objective data for inputs such as morale are difficult to obtain, we argue that including such significant factors is important to reflect modern land warfare in a meaningful way. Consequently, some input parameters may require subjective input data and sensitivity analysis around key estimates.

\section{METHOD}

SD has been used to model different aspects of land combat systems. This includes modelling the effects of engagement and attrition, the movement and formation of forces, the dynamics between opposing forces, the logistics and maintenance supplying battlefields, communication, insurgency operations as well as human factors affecting operations and forces. Despite these applications, SD combat modelling studies have not yet 
evolved into a mature line of research. For more detailed review, readers are referred to Cunico et al. (2021). In addition, no existing SD models focus at the intermediate level of aggregation between the high-fidelity and highly aggregated models discussed above.

The standard SD model building process includes five stages (Sterman, 2000): (i) problem articulation involves defining the specific dynamic issue to be addressed and identifying behaviour over time graphs of key variables, (ii) model conceptualisation includes formulating a dynamic hypothesis about the set of causal relationships responsible for driving the dynamics of the key variables, (iii) converting the conceptual model into a quantitative system dynamics simulation model, (iv) model testing and validation including Subject Matter Expert interviews/workshops and numerical data collection, and (v) what-if simulations and policy analysis to explore the dynamic consequences of different strategies and policies. This paper focuses on the model conceptualisation phase of building a qualitative causal model of combined arms tactical Land warfare.

Over a period of several months, DSTG subject matter experts on land combat and two SD modelling experts discussed the problem definition and model conceptualisation of the Baseline model. Throughout these discussions, the team understood that the Baseline model would need to be formulated so that it could later be expanded and/or adapted to examine different combat team force designs, incorporating relevant new and emerging combat technologies, and explore the scenario space of different operations within different operating environments and threat levels, without requiring the long lead times associated with building a high-fidelity, detailed model from scratch each time. For example, initially we have used a simplified 2 X 2 scenario space defined by the type of Red Force threat (i.e., Conventional Peer or Hybrid Force) and the type of operating environment (i.e., Complex or Open terrain). Although, the expectation is that eventually we will represent the scenario space with more detail, firstly the Baseline model must be conceptualised to enable rapid expansion or adaptation to represent any of these four scenarios and to include different potential force designs including combat technologies and new ways of fighting.

\section{CONCEPTUAL MODEL}

The team drew heavily on prior DSTG work (Kempt et al., 2021), Army doctrine, and prior published combat team models to identify an initial list of key variables to include in the Baseline model. To represent a combat team, we decided to include force element variables such as Personnel and Vehicles. Our discussions also highlighted the importance of including many qualitative variables in the Baseline model to represent contemporary conflict. For example, we decided the model must include Will to Fight, Situation Awareness, Freedom of Action, Psychological/Information Operations Effects, Morale, Level of Connectivity, and Synchronisation of Force Effects. Including these variables extends the boundary of most land combat models that focus primarily on attrition of Red and Blue forces. Undermining an adversary's Will to Fight can potentially end a conflict more rapidly and with fewer casualties than engaging in a war of attrition.

A high-level sector diagram representing the key combat system components included in the Baseline model is shown in Figure 1. A bullet list of some of key variables is shown within each sector. The Combat Team Force Elements \& Effects sector in the top left includes vehicles, personnel, operational tempo, vehicle direct fires and personnel direct fires. These force elements and effects are represented for both the Blue and Red forces as indicated by the blue colouring and label in the front and the red colouring and label for a mirror image of this sector in the background. The Red force adversary could be a conventional peer or a hybrid force. The sector diagram shows the Blue force and Red force represented at the same level of detail across all sectors.

The Command \& Control sector in the bottom left includes situation awareness (actual and perceived), information foraging, command system effectiveness, level of connectedness (i.e., communications) and synchronisation of force effects. High synchronisation enhances the impact of the force effects. The Attrition sector in the upper middle of the diagram includes lethality (by force effect), vehicle attrition, and personnel attrition. The Sustainment sector in the top right includes replenishment of ammunition, petrol, oils and lubricants, repairs and maintenance and reinforcements of vehicles and personnel. The Non-Organic Fires \& Theatre Effects sector in the lower middle of the diagram includes indirect fires (from Army, Air or Navy), psychological operations/information operations effects and cyber/electronic warfare effects. The Will to Fight sector in the bottom right includes morale, cohesion, and the level of will to fight. The Operating Environment sector on the left side of the diagram includes physical terrain complexity and human terrain complexity. This sector is exactly the same for both the Blue and Red forces.

The black arrows drawn between sectors highlight only the most important causal connections. For example, a higher operation tempo in the Combat Team Force Elements \& Effects sector increases the demand for services from the Sustainment sector (i.e., the arrow from Combat Team Force Elements \& Effects to Sustainment). The provision of those sustainment services enables the vehicles and personnel to continue 
combat operations (i.e., the arrow from Sustainment to Combat Team Force Elements \& Effects).

As part of the model conceptualisation, the team also developed an incomplete hybrid stock \& flow diagram.

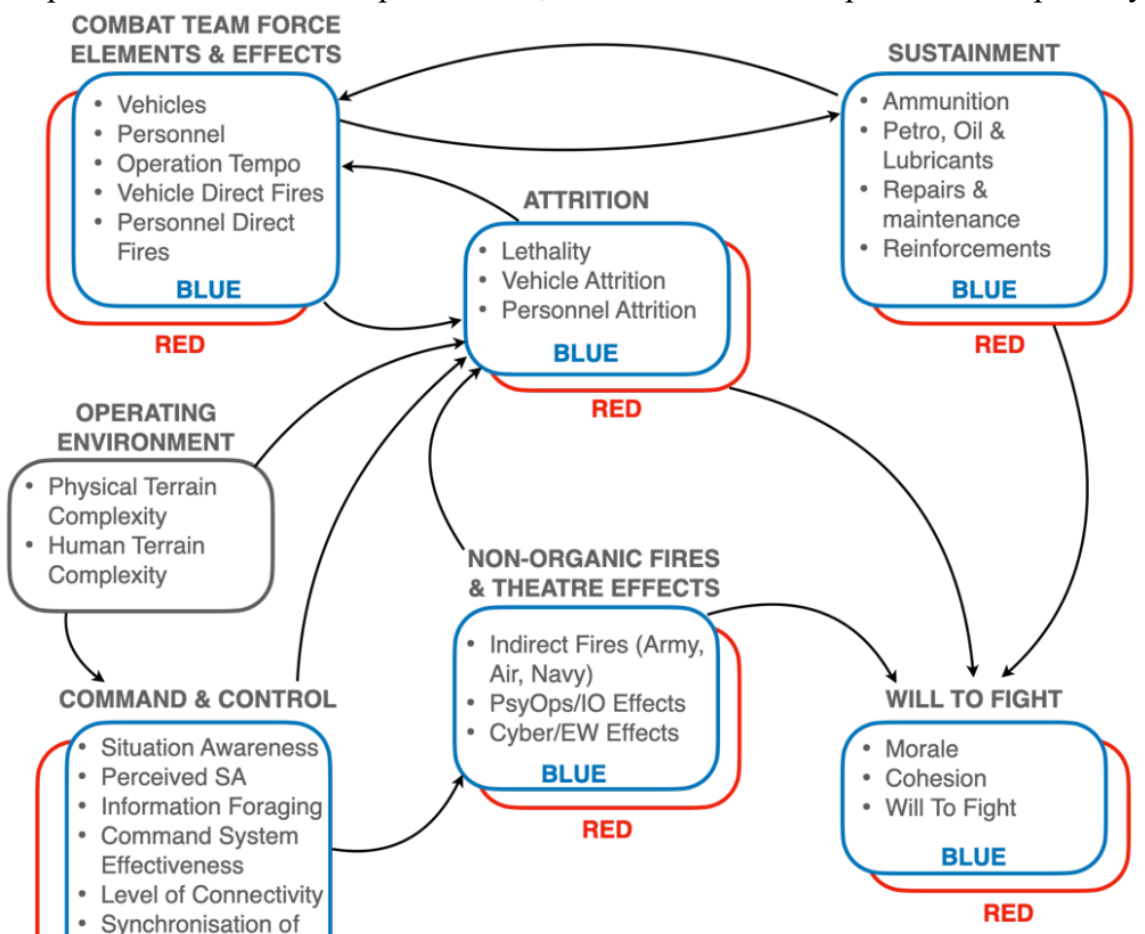

Figure 1. Sector diagram of combat system components included in Baseline model Shown in Figure 2, this artefact served as an initial, preliminary conceptual model that included more detail than Figure 1, but remained fairly high-level (e.g., limited detail of combat force elements and effects). Again, the team drew heavily on the causal diagrams in Kempt et al. (2021). The initial version was drawn on a whiteboard and was subsequently rebuilt using Vensim simulation software

(https://vensim.com/). For visual simplicity, the diagram does not include the full two-sided (i.e., Blue Force and Red Force) combat model. Also, this preliminary diagram includes points noted during the team discussion, including two stocks considered important to include later. We emphasise that the model conceptualisation continued evolving as we drew more detailed hybrid stock and flow diagrams.

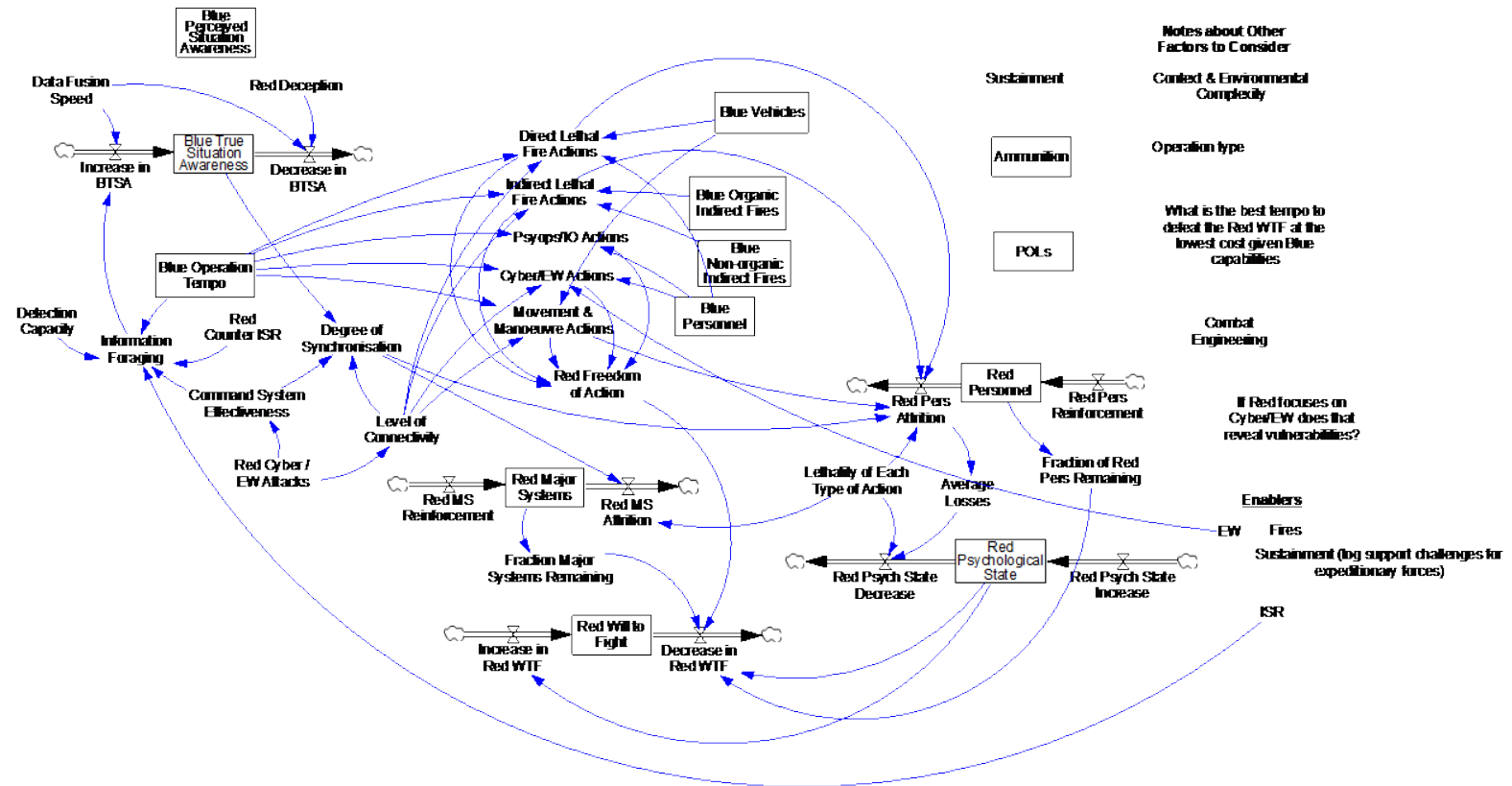

Figure 2. Partial Baseline conceptual model as a hybrid stock and flow diagram

Next, the team converted a small piece of the high-level hybrid diagram into a more detailed stock and flow structure closer to the structure that will be required for the simulation model. We started by focusing on the Vehicle Direct Lethal Fires since that part of the system is well understood. See Figure 3 below. 


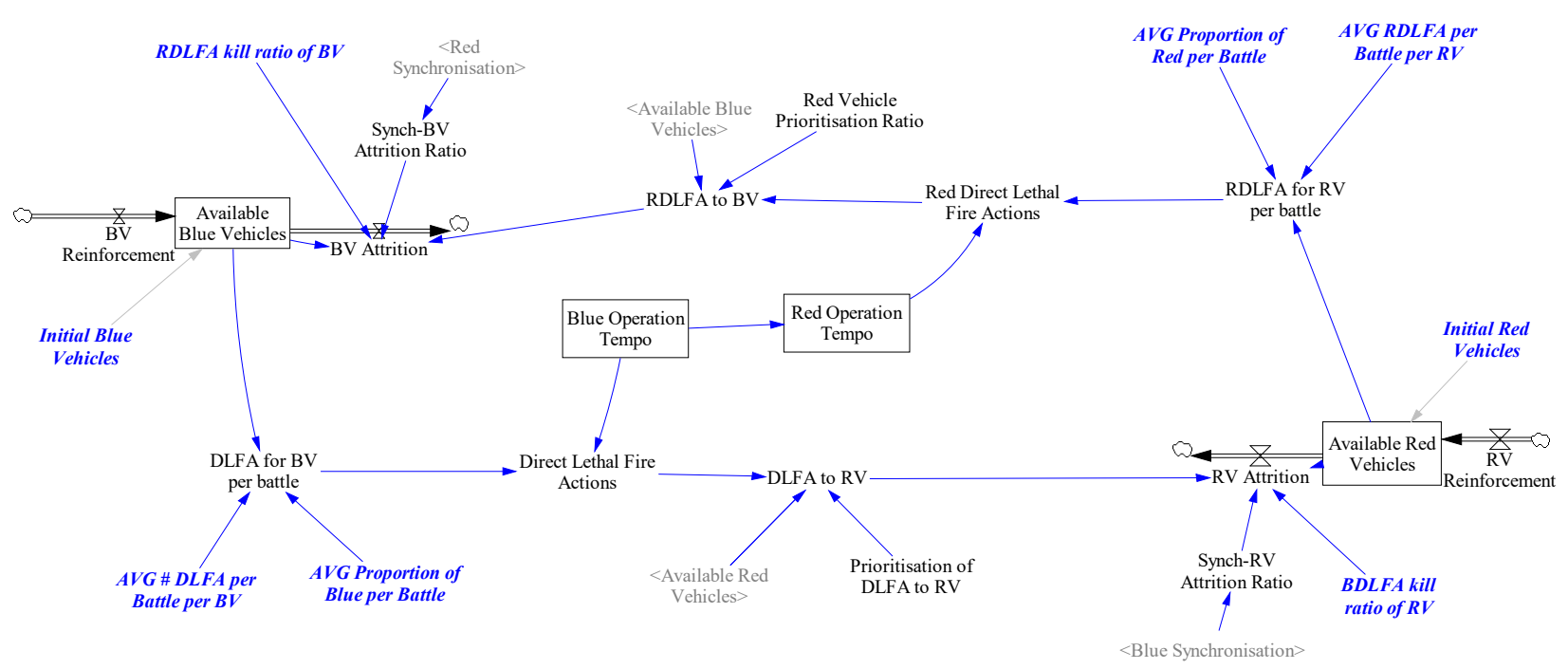

Figure 3. Stock and flow diagram of Vehicle Direct Lethal Fires

We decided to define variables in the model in terms of a time constant of one week. For example, we defined Blue Vehicle Direct Lethal Fire Actions (DLFA) in units of: \# Fire Actions per Week. Blue Vehicle DLFA is determined by Blue Operation Tempo [measured in Battles per Week] multiplied by Average \# Fire Actions per Battle. However, this desired number of fire actions per week may be constrained by the number of Available Blue Vehicles at any given time, and the AVG Proportion of Blue per Battle which indicates how much of the blue force will be engaged in each battle. DLFA increases the Attrition Rate of Red Vehicles based on the Lethality of Blue Direct Fires (BDLFA kill ratio of RV) and the extent to which the Direct Fires were synchronised (i.e., reduced redundancy of DLFA). Synchronisation is currently modelled by introducing a penalty multiplier to the lethality of Blue where low synchronization means lower lethality rates. This model is mirrored for Red and replicates the classical Lanchester Square Law (Artelli and Deckro, 2008). This stock and flow diagram will continue to evolve as the team adds more detail and formulates the causal relationships into precise equations. For example, further work on the Direct Lethal Fires component will incorporate Command \& Control elements. Shadow variables in Figure 3 arise from other parts of the Baseline model.

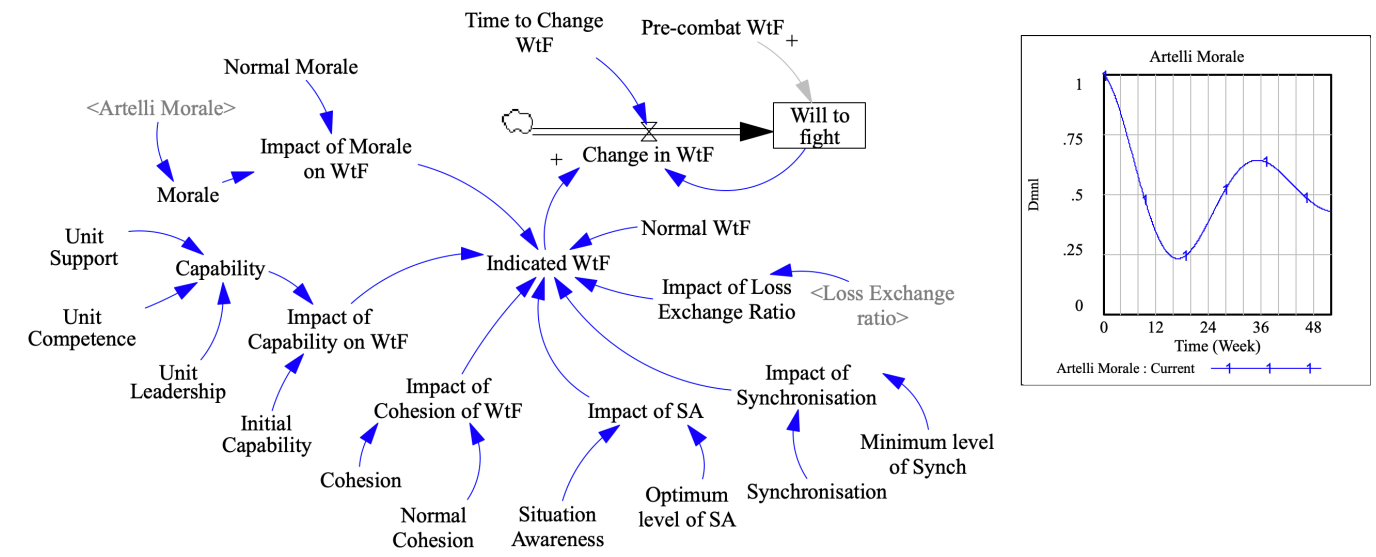

Figure 4. Will to Fight (WtF) preliminary stock and flow diagram

The team also elaborated the stock and flow structure for Will to Fight (WtF). Prior research identified many factors that impact WtF including: the unit's pre-combat state, combat state factors, adversary factors and other 'exogenous' factors (Connable et al. 2018)(Artelli et al. 2010; Bester and Stanz, 2007). The team drew from these studies to identify the most influential factors that drive WtF to include in the Baseline model. Figure 4 shows this preliminary $\mathrm{WtF}$ conceptualisation that includes the causal impacts on $\mathrm{WtF}$ of capability, cohesion, morale, Blue and Red loss exchange ratio (i.e., relative losses of soldiers and systems), situation awareness and synchronization. A specific test input for morale (see the graph in Figure 4), shows the evolution Artelli et al. (2010) identified for a typical unit's morale over the course of a 52 week deployment; morale is defined as the unit's mental and emotional condition. Research finds that morale influences combat readiness (Bester and 
Stanz, 2007) and combat effectiveness (Artelli et al., 2010). Many factors included in Figure 4 will become endogenous variables once the $\mathrm{WtF}$ sector becomes integrated with other sectors in the overall Baseline model.

We will continue to develop the full conceptual model to include stock and flow diagrams for other action types (e.g., indirect fires, information operations (IO), cyber and electromagnetic activities (CEMA), force elements (personnel, and indirect systems) and for all other sectors in the Baseline model (Figure 2).

\section{DISCUSSION AND NEXT STEPS}

Our longer term aims in developing effects-based tactical land warfare models include enabling:

- Agile analysis - medium-fidelity so that a range of combat teams can be assessed across the scenario space rapidly.

- $\quad$ Dynamic analysis - to understand, compare and validate the evolution of key parameters and operational performance of combat teams over time.

- Incorporation of quantitative \& qualitative variables - to represent all key variables and parameters

- Causal representation - to understand the interdependencies between key variables.

- Decision support - evidence base to support decisions on: research investment priorities, force designs and future capability needs, and to guide additional evaluation (e.g. in closed loop simulation).

We plan to use the models to inform which technologies are likely to have significant impact on a future combat team overall performance. For example, are investments in improving sensor technologies likely to have a greater impact on combat team effectiveness than (say) artillery systems or cyber effectors? The models will also enable comparison of the relative effectiveness of proposed future combat teams across a range of scenarios. For example, combat teams enabled by significant robotics and autonomous systems may perform well in part of the scenario space, whereas combat teams with advanced low signature communications - but designed to be light and fight dispersed - may perform well in other parts. For a given combat team design, the models should also examine strengths/weakness and in which part of the scenario space they can operate to an acceptable level of effectiveness (i.e., what is its feasible scenario space?) (Bowden, Pincombe, and Williams, 2015). For example, some combat team designs may perform well across a range of environments (e.g., urban, jungle etc.) but struggle to adapt to different adversary tactics (e.g., conventional conflict versus insurgency).

Keeping these aims in mind, the team is currently investigating relevant reports and publications where additional knowledge is required to understand how to best represent different model variables/concepts (e.g., Will to Fight, CEMA, Situation Awareness, Information Foraging, Synchronisation). Also, the team is in the process of converting the remaining pieces of the high-level conceptual model into more detailed stock and flow diagrams (e.g., Psyops/IO Actions, Indirect Lethal Fire Actions). In addition, we will consult with SMEs knowledgeable about specific aspects of the conceptual model (e.g., CEMA Actions) to refine parts of the conceptual model. After elaborating and refining all components of the conceptual model, the team will convert each stock and flow diagram - each diagram representing a small piece of the overall conceptual model - into a quantitative system dynamics simulation model. Where needed, we will refer to the published literature for ideas on units of measure and equation formulations. The team also will identify data requirements for model development, calibration and testing, and collect the data. Each small model will be tested upon completion to ensure robust equation formulations, and to ensure that the small model exhibits dynamic behaviour consistent with expectations and/or data, and structural assumptions consistent with the real system. After testing each small component, it will be added to the Baseline simulation model until eventually all components have been assembled. Next, the full model must go through testing and validation.

Once the Baseline model has been completed, the next step involves extending the model to incorporate the effects of emerging technologies. For example, adding the effects of autonomous systems will enable exploration of operational effectiveness of that technology across different operating environments. A key advantage of the SD modelling approach is the ability to represent both qualitative and quantitative variables and parameters. Considering the semi-autonomous combat team concept (Sawers and Tang, 2020), humanmachine teaming (HUM-T) is paramount where robotic platforms, artificial intelligence and sensor suites, integrated with an information cloud, improve situation awareness of the future soldier, increase combat mass and reduce risk of injury. The realization of HUM-T will require the integration of numerous technologies with humans and the commensurate technical challenges including reliability and vulnerabilities including cyberattack (Ryan, 2018). While many of these aspects can be quantitatively modelled, other challenges which could undermine performance, are more qualitative including trust, ethics, and legal constraints.

While SD provides many benefits, we envisage eventually evolving the model architecture into a multi-method approach where models from other simulation paradigms are included; for example, discrete event simulations 
may investigate a particular sector in detail and then provide inputs into the SD model or agent based models may explore new warfighting concepts to inform updates to the SD model representation. The approach outlined here provides opportunities to incorporate all these aspects into the planned simulation models and then run 'what if' analyses to explore the sensitivity on combat team effectiveness.

\section{REFERENCES}

Anderson Jr EG. 2011. A dynamic model of counterinsurgency policy including the effects of intelligence, public security, popular support, and insurgent experience. System Dynamics Review 27(2): 111-141.

Artelli MJ, RF Deckro. 2008. Modeling the Lanchester Laws with System Dynamics. The Journal of Defense Modeling and Simulation: Applications, Methodology, Technology 5: 1-20.

Artelli MJ, RF Deckro, DJ Zalewski, SE Leach, MB Perry. 2010. A control theory model of deployed soldiers' morale. Int. J. Operational Research 7(1).

Balogh, G Harless 2003. An Overview of the COMBAT XXI Simulation Model: A Model for the Analysis of Land and Amphibious Warfare. In Proceedings of the Proc. of 71st Military Operations Research Society Symposium. USMCB Quantico, VA.

Bester PC, KJ Stanz. 2007. The Conceptualisation and Measurement of Combat Readiness for Peace-Support Operations - An Exploratory Study. SA Journal of Industrial Psychology 33(3): 68-78.

Bowden FDJ, B Pincombe, PB Williams 2015. Feasible Scenario Spaces: a new way of measuring capability impacts. In Weber, T., McPhee, M.J. and Anderssen, R.S. (eds) MODSIM2015, 21st International Congress on Modelling and Simulation. Modelling and Simulation Society of Australia and New Zealand, December 2015, pp. 836-842.

Bowley D, T Castles, A Ryan. 2003. Constructing a SUITE of Analytical Tools: A Case Study of Military Experimentation. ASOR BULLETIN 22(4).

Chau W, A Gill, D Grieger 2017. Using Combat Simulation and Sensitivity Analysis to Support Evaluation of Land Combat Vehicle. In Syme, G., Hatton MacDonald, D., Fulton, B. and Piantadosi, J. (eds) MODSIM2017, 22nd International Congress on Modelling and Simulation. Modelling and Simulation Society of Australia and New Zealand, December 2017, pp. 536-542.

Connable B, MJ McNerney, W Marcellino, A Frank, H Hargrove, MN Posard, SR Zimmerman, N Lander 2018. Will to Fight: Analyzing, Modeling, and Simulating the Will to Fight of Military Units. RAND Corporation.

Cunico G, S Elsawah, S Gary, T Cao, L Kosowski, M Richmond 2021. System dynamics applications for defence combat modelling: preliminary insights from a literature exploration. In Proceedings of the 21 st International Congress on Modelling and Simulation.

Finlay L 2013. Closing the Loop with the Close Action Environment. In Piantadosi, J., Anderssen, R.S. and Boland J. (eds) MODSIM2013, 20th International Congress on Modelling and Simulation. Modelling and Simulation Society of Australia and New Zealand, December 2013, pp. 1005-1011.

Gach T, M Sanders 2015. Advanced Warfighting Simulation (AWARS). U.S. Department of Defense, Retrieved from: https://www.trac.army.mil/20150910Awars.pdf.

Gaidow S, L Kosowski, J O’Hanlon. 2021. Influence Diagrams for Modelling Tactical Warfighting Functions. DSTG-TR-3802, Commonwealth of Australia.

Kempt N, L Kosowski, G Weinberg. 2021. A Preliminary Causal Model for Understanding the Land Close Combat Ecosystem. DSTG-TN-2155, Commonwealth of Australia.

Nguyen M-T, T Cao. 2019. A multi-method approach to evaluate land combat vehicle system. International Journal of Applied Decision Sciences 12(4): 337-360.

Pincombe A, B Pincombe, CEM Pearce. 2010a. A simple battle model with explanatory power. ANZIAM Journal 51(EMAC2009): C497-C511.

Pincombe AH, BM Pincombe, CEM Pearce. 2010b. Putting the art before the force. ANZIAM Journal 51(EMAC2009): C482-C496.

Ryan M. 2018. Human-machine Teaming for Future Ground Forces. Center for Strategic and Budgetary Assessments (CSBA).

Sawers M, K Tang. 2020. Semi-Autonomous Combat Team Dismounted Infantry 2030 Concept. DST-GroupGD-1068.

Speight, D Rowland. 2006. Modelling The Rural Infantry Battle: Overall Structure and a Basic Representation of the Approach Battle. Military Operations Research 11(1): 5-26.

Sterman JD. 2000. Business Dynamics: Systems Thinking and Modeling for a Complex World. Irwin/McGrawHill, New York.

Veldhuis GA, P van Scheepstal, E Rouwette, T Logtens. 2015. Collaborative problem structuring using MARVEL. EURO Journal on Decision Processes 3(3-4): 249-273. 